\title{
Non-Linear Oberbeck Convection in Chiral Fluid through a Vertical Permeable Channel in the Presence of a Transverse Magnetic Field
}

\author{
N. Rudraiah ${ }^{1 \dagger}$, B. M. Rajaprakash ${ }^{2}$ and Nagaraju ${ }^{3}$ \\ ${ }^{1}$ National Research Institute for Applied Mathematics, Bangalore-560 070, India \\ ${ }^{2}$ Department of Mechanical Engineering, University Visveswaraya College of Engineering K.R. Circle, Bangalore \\ 560001, India \\ †Corresponding Author Email: rudraiahn@hotmail.com
}

(Received March 10, 2012; accepted March 7, 2013)

\begin{abstract}
Nonlinear oberbeck convection in a chiral incompressible Boussinesq fluid flowing through a vertical channel bounded by rigid permeable isothermal boundaries kept at different temperatures is investigated in this paper in the presence of a uniform transverse magnetic field, under the influence of viscous dissipation. The nonlinear-coupled momentum and energy equations are solved analytically using a regular perturbation method valid for small values of buoyancy parameter $\mathrm{N}$. To validate the results obtained from the analytical solutions of the non-linear equations, which are also solved numerically using a finite difference method supplemented with the successive over recreation (SOR) technique. The velocity, temperature, skin friction, mass flow rate and the rate of heat transfer are computed for various values of electromagnetic thermal number $\mathrm{W}_{\mathrm{emt}}$, buoyancy parameter $\mathrm{N}$, and suction Reynolds Number $\mathrm{R}_{\mathrm{e}}$. The results obtained are represented graphically and found that an increase in $\mathrm{W}_{\mathrm{emt}}$, increases the velocity and temperature. Physically, we attributes this to setting up of small scale turbulence by magnetic filed. The effect of perturbation parameter $\mathrm{N}$ is shown to increases the skin friction, heat transfer and the mass flow rate.
\end{abstract}

Keywords: Chirality, Buoyancy parameter, Convective current, Lorentz force

\section{NOMENCLATURE}

$B_{0}$ applied magnetic field

$C_{p} \quad$ specific heat capacity

$\vec{D} \quad$ dielectric field

$\vec{H} \quad$ magnetic field

$\vec{E} \quad$ electric field

$\vec{g} \quad$ acceleration due to gravity

b width of the channel

$\vec{J} \quad$ convective current density

$k$ thermal conductivity

$w_{\text {emt }}$ electromagnetic thermal number

$N$ buoyancy parameter

$\mu$ magnetic permeability

$\phi$ viscous dissipation
$P e$ Peclet number

p pressure

$\vec{q}=(u, v)$ velocity components in $x$ and $y$ directions

$R_{1}$ viscosity variation parameter

Re Reynolds number

$T$ absolute temperature

$\beta_{T}$ coefficient of thermal expansion of density

$\varepsilon \quad$ dielectric constant

$\gamma$ chirality coefficient

$v$ kinematic viscosity

$\mu_{f}$ viscosity of fluid

$\rho_{e}$ distribution of electric charge density

$\theta$ non dimensional temperature 
$\rho$ density of fluid

\section{INTRODUCTION}

This Mechanical, electrical and electronic devices give off heat as a byproduct of power consumption when a device is being used. Fans and regular fluids are usually used to improve the cooling processes, but they increase the device weight, size and bulk. Now a day, the industries continue to churn out miniature and portable devices. For this purpose, manufactures are challenged to find new ways to combat the persistence problem of thermal management. In addition, miniaturization has an increasing importance in many fields in the present day modern technological world. In the current industrial environment, there is a growing need to miniature devices, which are capable of delivering more power. There is of great concern for ensuring safety and integrity of contacting components which are to be enabled to resist fretting, wear, erosion, galling, scuffing and damage with thermal management application. When reduced to sub-millimeter size, the integrity of the material typically used for cooling structure breaks down, materials like silicon become very brittle and easily shatter, while metallic structures used become bendable and weak. Therefore, there is an urgent need of suitable materials to overcome the above deficiencies. At present, there is no doubt that nano and smart materials are becoming popular for use in overcoming the above deficiencies, but they continue to be aggravated by heat. Apart from using these novel materials, for the first time in the literature an attempt is made to use a chiral material (solid or fluid) for the above purpose. The works of Arago (1811), Boit (1812), Jaggard et al. (1979) and Engheta and Micklson (1982) reveal that solid chiral materials have been used to manufacture devices like antennas. However, much attention has not been given to chiral fluids like turpentine, sugarcane solution used in sugar industries, body fluids used in designing artificial organs, and so on.

A chiral material is defined as the one which cannot be brought into congruence with its mirror image by any amount of translation and rotation, and therefore having the property of handedness, either a left-handed or a right- handed, Rudraih et al. (1977). Since most of the chiral fluids are volatile in nature, they can be efficiently used for cooling purposes involving effective heat transfer. This heat transfer may also be due to density changes with temperature, when the temperature gradient is aligned to the direction of gravity, called natural convection, also called RayleighBenard convection. However, if the temperature gradient is maintained perpendicular to gravity, fluid movement arises instantaneously, due to the generation of vortices at the boundaries see Rudraiah et al. (1977), a phenomenon called Oberbeck convection. The Oberbeck convection in ordinary fluid and fluid saturated porous media, have been investigated by Rudraiah and Nagaraju (1977) and Rudraiah et al. (1982). But, to our knowledge, much attention has not been given to the study of Oberbeck convection in chiral fluids through a vertical channel in spite of their importance in many practical applications like in purification of sugar cane solution in sugar industries,
0 reference quantity

in the biomedical engineering in the study of controlling stonosis in trachea (a wind pipe) formed by accumulation of tiny aerosols and so on, The study of it, in a chiral incompressible Boussinesq fluid, incorporating chirality in electromagnetic constitutive equations in the presence of a transverse magnetic field, is the objective of this paper.

To achieve the above objective of this paper, the required basic equations supplemented with the Maxwell field equations, continuity of charges and the constitutive equations for chirality are given in section 2. Analytical solutions of coupled nonlinear momentum and energy equations are obtained in section 3 using the regular perturbation technique. To know the validity of an analytical solution, numerical solutions are also obtained in section 4 using the finite difference scheme. The skin friction, heat transfer and the mass flow rate are obtained in section 5 and the important conclusions are drawn in the final section.

\section{MATHEMATICAL Formulation}

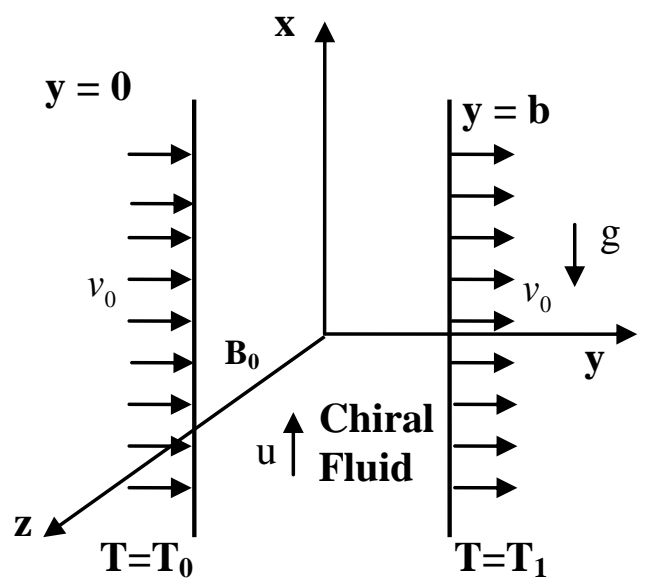

Fig. 1. Physical configuration

The physical configuration, considered in this paper, consists of an infinite vertical channel bounded on both sides by rigid permeable isothermal boundaries of width $b$, kept at different temperatures as shown in Fig. 1. The temperatures of the hotter and cooler plates respectively are $T_{1}$ and $T_{2}$. A transverse magnetic field of uniform strength $B_{0}$ is applied in the $z$ - direction. For mathematical formulation, we consider the two dimensional motion with the $x$-axis along the vertical direction and the $y$-axis normal to it. Then the required basic equations for an Oberbeck-Boussinesq chiral fluid in the absence of displacement current and induced magnetic field because of negligible electrical conductivity are: 


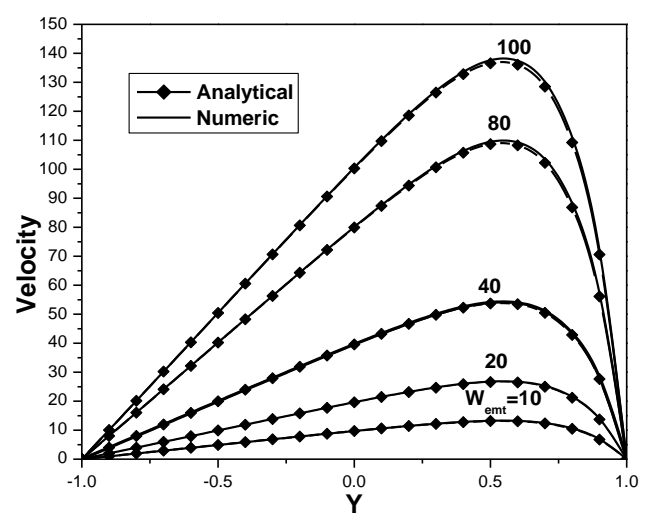

Fig. 2. Velocity profile for various values of $\mathrm{W}_{\mathrm{emt}}$ when $\mathrm{N}=0.01, \mathrm{R}_{\mathrm{e}}=5, \mathrm{P}_{\mathrm{e}}=7$

The conservation of Mass for an incompressible fluid

$\nabla \cdot \overrightarrow{\mathrm{q}}=0$

Equation of state for a Boussinesq fluid

$\rho=\rho_{0}\left(1-\beta_{T}\left(T-T_{0}\right)\right)$,

\section{Conservation of Momentum}

$\rho_{0} \frac{D \vec{q}}{D t}=-\nabla p+\rho \vec{g}+\mu_{f} \nabla^{2} \vec{q}+\vec{J} \times \vec{B}$

Conservation of Energy

$\rho_{0} c_{p} \frac{D T}{D t}=K \nabla^{2} T+\phi$

where $\frac{D}{D t}=\frac{\partial}{\partial t}+(\vec{q} \cdot \nabla), \quad \vec{q} \quad$ is the velocity with components $(u, v), p$ the pressure, $\vec{J}=\rho_{e} \vec{q}$, the convective current density, $\mu_{f}$ the viscosity of chiral fluid, $T$ the temperature, $K$ the thermal conductivity, $C_{p}$ the specific heat at constant pressure, $\rho_{0}$ the density of fluid at room temperature $T_{\boldsymbol{0}}, \beta_{T}$ the volumetric expansion coefficient and $\phi=\mu_{f}(\nabla \vec{q})^{2}$ is the viscous dissipation. We assume an uniform suction or injection velocity, $v_{0}$, at the boundaries. These equations have to be supplemented with the conservation of charges, $\rho_{e}$ for an incompressible fluid with convective current density given by

$$
\frac{\partial \rho_{e}}{\partial t}+(\vec{q} \cdot \nabla) \rho_{e}=0
$$

and the Maxwell's field equations

Gauss Law:

$$
\nabla \cdot \vec{D}=\rho_{e}
$$

Faraday's Law: $\quad \nabla \times \vec{E}=-\frac{\partial \vec{B}}{\partial t}$

Amperes Law:

$$
\nabla \times \vec{H}=\vec{J}=\rho_{e} \vec{q}
$$

$\nabla \cdot B=0$

A physical reason for considering only the convective current is that the chiral fluids like sugar cane solution, turpentine and so on have low relaxation frequencies where the convective current $\rho \vec{q}$ dominates over the displacement current $\partial \vec{D} / \partial t$. The constitutive equations for chiral fluid, following Rudraiah et al. (2000), Nader Engheta et al. (1989) and Lakhtakia et al. (1988), are

$$
\begin{aligned}
& \vec{D}=\varepsilon \vec{E}+i \gamma \vec{B} \\
& \vec{B}=\mu \vec{H}-\mu i \gamma \vec{E}
\end{aligned}
$$

where, $\gamma$ is the chirality coefficient, $\mu$ the magnetic permeability, $\varepsilon$ the dielectric constant, $\rho_{e}$ the density of distribution of charges.

In this paper we assume a fully developed and unidirectional flow in the $x$-direction, so that the velocity $u$ and temperature $T$ are independent of $x$ and $t$ and are function of $y$ only. In this case, the Lorentz force, $\vec{J} \times \vec{B}$, in the conservation of momentum with $\vec{q}=\left(u, v_{0}\right)$, is

$$
\vec{J} \times \vec{B}=\rho_{e}\left[v_{0} B_{0} \hat{i}-u B_{0} \hat{j}\right]
$$

Then the ' $x$ ' and ' $y$ ' components of the momentum Eq. (3) using the hydrostatic balance, $-\partial P / \partial y-\rho_{0} g=0$, steady and fully developed flow, take the form

$v_{0} \frac{\partial u}{\partial y}=g \beta_{T}\left(T-T_{0}\right)+v \frac{\partial^{2} u}{\partial y^{2}}+\frac{\rho_{e}}{\rho} v_{0} B_{0}$

$-\frac{\partial p}{\partial y}-\rho_{e} B_{0} u=0$

The energy equation in the presence of a viscous dissipation is

$v_{0} \frac{\partial T}{\partial y}=\kappa \frac{\partial^{2} T}{\partial y^{2}}+\frac{v}{C_{p}}\left(\frac{\partial u}{\partial y}\right)^{2}$

Making these equations dimensionless, using the scales $b$ for length, $\varepsilon V / b^{2}$ for charge density $\rho_{e}$, $g \beta b^{2}\left(T_{1}-T_{0}\right) / v \quad$ for velocity, $\quad \theta=\left(T-T_{0}\right) / \Delta T$ for temperature, $\Delta T=T_{1}-T_{0}, T_{0}$ the source temperature, $T_{1}$ the boundary temperature, $v$ the kinematic viscosity and after simplification we get

$$
\frac{\partial^{2} u}{\partial y^{2}}-\operatorname{Re} \frac{\partial u}{\partial y}+\theta+W_{e m t}=0
$$

$$
\frac{\partial^{2} \theta}{\partial y^{2}}-P e \frac{\partial \theta}{\partial y}+N\left(\frac{\partial u}{\partial y}\right)^{2}=0
$$

The solenoidal property of magnetic field 
where $\quad W_{e m t}=\rho_{e} \varepsilon_{0} V B_{0} v_{0} / \rho_{0} g \beta b^{2}\left(T_{1}-T_{0}\right) \quad$ is the electromagnetic thermal number, $N=\rho_{0} g^{2} \beta^{2}\left(T_{1}-T_{0}\right) b^{4} / K v$ the buoyancy parameter, $R e=v_{0} b / v$ suction Reynolds number, and $P e=v_{0} b / \kappa$ Peclet Number. We solve the above equations both analytically and numerically using the no-slip conditions on velocity and isothermal conditions on temperature. In dimensionless form these boundary conditions are:

$u=0$ at $y= \pm 1, \quad \theta=1$ at $y=1, \quad \theta=-1$ at $y=-1$

\section{ANALYTICAL SOLUTIONS}

Equations (16) and (17) are the coupled non-linear momentum and energy equations. Analytical solutions of which are obtained using Regular Perturbation technique with buoyancy parameter $N \quad(<<1)$ as perturbation parameter. In this technique, we compute $\mathrm{u}$ and $\theta$ in the series form given by.

$u=u_{0}+N u_{1}+N^{2} u_{2}+$

$\theta=\theta_{0}+N \theta_{1}+N^{2} \theta_{2}+$

Substituting Eq. (19) and Eq. (20) into Eq. (16) and Eq. (17) and equating the coefficients of like powers of $N$ to zero restricting the terms only up to the order unity because $N<<1$, we get the following boundary value problems

Zeroth order equations:

$$
\begin{aligned}
& \frac{d^{2} u_{0}}{d y^{2}}-\operatorname{Re} \frac{d u_{0}}{d y}+\theta_{0}+W_{e m t}=0 \\
& \frac{d^{2} \theta_{0}}{d y^{2}}-P e \frac{d \theta_{0}}{d y}=0
\end{aligned}
$$

The corresponding boundary conditions obtained from Eq. (18) using Eq. (19) and Eq. (20) are:

$$
u_{0}=0 \text { at } \quad y= \pm 1, \quad \theta_{0}=1 \text { at } y=1, \quad \theta_{0}=-1 \text { at } y=-1
$$

Then, the solutions of Eq. (21) and Eq. (22) satisfying the boundary conditions Eq. (23) are:

$$
\begin{aligned}
& u_{0}=A_{4} e^{P e y}+A_{3} y+A_{6}+A_{5} e^{\mathrm{Re} y} \\
& \theta_{0}=-A_{1}+A_{2} e^{P e y}
\end{aligned}
$$

The first order equations are:

$$
\begin{aligned}
& \frac{d^{2} u_{1}}{d y^{2}}-\operatorname{Re} \frac{d u_{1}}{d y}+\theta_{1}+W_{e m t}=0 \\
& \frac{d^{2} \theta_{1}}{d y^{2}}-P_{e} \frac{d \theta_{1}}{d y}+\left(\frac{d u_{0}}{d y}\right)^{2}=0
\end{aligned}
$$

and the corresponding boundary conditions obtained from Eq. (18) are:

$$
u_{1}=0 \text { at } y= \pm 1, \theta_{1}=0 \text { at } y=1, \theta_{1}=0 \text { at } y=-1
$$

The solutions of Eq. (27) and Eq. (28) satisfying the boundary conditions Eq. (28), are:

$$
\begin{aligned}
& u_{1}=\left(A_{3}{ }^{2} / a_{1}\right)\left(a_{14}+2 e^{\operatorname{Re} y} a_{12}+y+y^{2}\right)+A_{4}{ }^{2}\left(a_{2}+\left(e^{2 P e y} / a_{3}\right)\right)+ \\
& \left(A_{7} / \operatorname{Re}\right)\left(e^{\operatorname{Re} y}\left(-y+a_{11}\right)-a_{12}\right)+A_{8}\left(a_{4}+\left(e^{(P e+\operatorname{Re}) y} / a_{14}\right)-e^{\operatorname{Re}(1+y)} a_{5}\right) \\
& +\left(A_{9} / \operatorname{Re}^{2}\right)\left(e^{\operatorname{Re} y} a_{12}-a_{12} y\right)+\left(A_{10} / a_{15}\right)\left(a_{12}-a_{11}-e^{\mathrm{Re}} a_{12} a_{13}\right)+ \\
& \left(A_{11} / \operatorname{Re}\right)\left(y+a_{11}-e^{\operatorname{Re}} a_{12}\right)+\left(A_{3} A_{4} / a_{16}\right)\left(y e^{P e y} a_{6}+e^{\operatorname{Re} y} a_{7}+a_{8}\right)
\end{aligned}
$$

$$
\begin{aligned}
\theta_{1}= & -\left(A_{3}^{2} / P e\right) Y+2 A_{3} A_{4} Y e^{P e Y}+A_{7} e^{\mathrm{Re} Y}-\left(A_{4}^{2} / 2\right) e^{2 P e Y} \\
& +A_{8} e^{(P e+\mathrm{Re}) Y}+A_{9} e^{2 \operatorname{Re} Y}+\left(A_{10} / a_{13}\right) e^{P e Y}+A_{11}
\end{aligned}
$$

where the coefficients $A_{i}(i=1 \ldots . .11)$ are given in the appendix to this paper.

\section{NUMERICAL SOLUTIONS}

The analytical solutions Eq. (29) and Eq. (30) obtained in section 3, using a regular perturbation technique with $\mathrm{N}$ as the perturbation parameter is valid only for small values of the parameter $\mathrm{N}$. To know the validity of these solutions we solve them numerically in this section using a finite difference technique. The finite difference form of Eq. (16) and Eq. (17) using the central difference scheme with 21 mesh points with step (h) size 0.1 are:

$$
\mathrm{L}_{l}=\mathrm{u}_{\mathrm{j}}=\frac{1}{2}\left[\mathrm{u}_{\mathrm{j}+1}+\mathrm{u}_{\mathrm{j}-1}-(\operatorname{Reh}) / 2\left(\mathrm{u}_{\mathrm{j}+1}-\mathrm{u}_{\mathrm{j}-1}\right)+\theta_{\mathrm{j}} \mathrm{h}^{2}+\mathrm{W}_{\mathrm{emt}} \mathrm{h}^{2}\right]
$$

$\mathrm{L}_{2}=\theta_{\mathrm{j}}=\frac{1}{2}\left[\theta_{\mathrm{j}+1}+\theta_{\mathrm{j}-1}-(\right.$ Peh $\left.) / 2\left(\theta_{\mathrm{j}+1}-\theta_{\mathrm{j}-1}\right)+\frac{\mathrm{N}}{4}\left(\mathrm{u}_{\mathrm{j}+1}-\mathrm{u}_{\mathrm{j}-1}\right)^{2}\right]$

where the step length $h=0.1$ and the suffix j represent the mesh point which varies from 1 to20. Eq. (31) and Eq. (32) give two implicit equations in $u_{j}$ and $\theta_{j}$ represented by $L_{l}$ and $L_{2}$ respectively. They generate, separately, the system of algebraic equations for $u_{j-1}, u_{j}, u_{j+l}$ and $\theta_{j-1}, \theta_{j}, \theta_{j+l}$ resulting in tridiagonal coefficient matrices. These equations are solved using the method of successive over relaxation (SOR) technique. In this technique we choose the initial values of $u_{j}$ and $\theta_{j}$ to be zero. This automatically corrects in the iteration procedure. In this relaxation method the scheme for $k^{\text {th }}$ iteration at $j^{\text {th }}$ mesh point is

$\mathrm{u}_{\mathrm{j}}^{\mathrm{k}}=\omega\left[\mathrm{u}_{\mathrm{j}}^{\mathrm{k}}\right]_{\mathrm{L}_{1}}+(1+\omega) \mathrm{u}_{\mathrm{j}}^{\mathrm{k}-1}$ and $\theta_{\mathrm{j}}^{\mathrm{k}}=\omega\left[\theta_{\mathrm{j}}^{\mathrm{k}}\right]_{\mathrm{L}_{2}}+(1+\omega) \theta_{\mathrm{j}}^{\mathrm{k}-1}$

where ' $\omega$ ' is the relaxation parameter. This translates Eq. (31) and Eq. (32) respectively to 


$$
\begin{aligned}
& \mathrm{u}_{\mathrm{j}}^{\mathrm{k}}=\frac{\mathrm{w}}{2}\left[\mathrm{u}_{\mathrm{j}+1}^{\mathrm{k}}+\mathrm{u}_{\mathrm{j}-1}^{\mathrm{k}}-(\operatorname{Reh}) / 2\left(\mathrm{u}_{\mathrm{j}+1}-\mathrm{u}_{\mathrm{j}-1}\right)+\theta_{\mathrm{j}} \mathrm{h}^{2}+\mathrm{W}_{\mathrm{ent}} \mathrm{xh}^{2}\right]+ \\
& \mathrm{w}\left[\mathrm{u}_{\mathrm{j}}^{\mathrm{k}}\right]_{\mathrm{L}_{1}}+(l+\mathrm{w}) \mathrm{u}_{\mathrm{j}}^{\mathrm{k}-1} \\
& \theta_{\mathrm{j}}^{\mathrm{k}}=\frac{\mathrm{w}}{2}\left[\theta_{\mathrm{j}+1}^{\mathrm{k}}+\theta_{\mathrm{j}-1}^{\mathrm{k}}-(\text { Peh }) / 2\left(\theta_{\mathrm{j}+1}-\theta_{\mathrm{j}-1}\right)+(\mathrm{N} / 4)\left(\mathrm{u}_{\mathrm{j}+1}-\mathrm{u}_{\mathrm{j}-1}\right)^{2}\right]+ \\
& \mathrm{w}\left[\theta_{\mathrm{j}}^{\mathrm{k}}\right]_{\mathrm{L}_{1}}+(1+\mathrm{w}) \theta_{\mathrm{j}}^{\mathrm{k}-1}
\end{aligned}
$$

where $y_{j}=y_{0}+j h$ with the initial guess $y_{j}=0$.

The boundary conditions for u given by Eq. (18) are incorporated as $\mathrm{u}_{0}=0$ and $\mathrm{u}_{20}=0$. Similarly we use Eq. (18) to obtain as $\theta_{Y=1}=1$ and $\theta_{Y=-1}=-1$ that is $\theta_{0}=1$ and $\theta_{20}=-1$. Then Eq. (34) and Eq. (35) are solved simultaneously from $k=0$ at each mesh $j$-point till the required order of accuracy $\left|\mathrm{u}_{\mathrm{j}}^{\mathrm{k}+1}-\mathrm{u}_{\mathrm{j}}^{\mathrm{k}}\right|<10^{-5}$ of iterative difference is achieved after comparison with the modified solution. The analytical and numerical results obtained are plotted graphically in Fig. 2 and Fig. 3 and the results are discussed in section 6 .

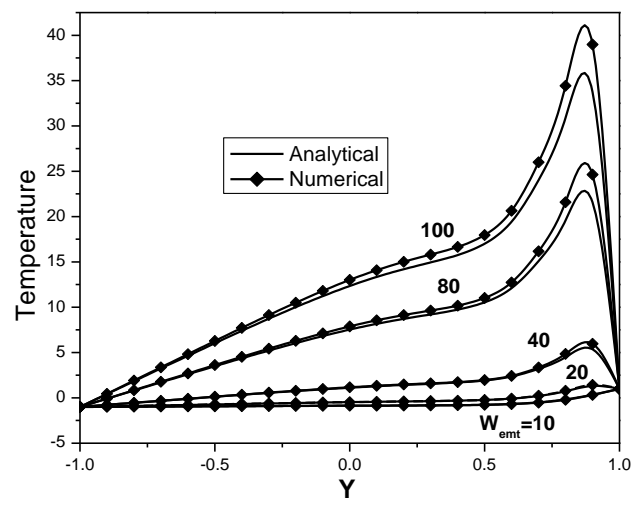

Fig. 3. Temperature profile for various values of $\mathrm{W}_{\mathrm{emt}}$ when $\mathrm{N}=0.01, \mathrm{Re}=5, \mathrm{Pe}=7$

\section{Skin Friction, RATE OF HEAT TranSFER AND MASS Flow RATE}

In many practical applications, involving separation of flow, it is advantages to know the skin friction, the rate of heat transfer and mass flow rate at the boundaries. The skin friction can be calculated from the shear stress ' $\tau$ ' at the walls, defined by

$$
\tau=\mu(\partial u / \partial y)_{y= \pm b}
$$

Making this dimensionless using, the scales $\rho g \beta b \Delta T$ for ' $\tau$ ', $g \beta b^{2}\left(T_{1}-T_{0}\right) / v$ for ' $u$ ' and ' $b$ ' for ' $y$ ', we get the dimensionless skin friction

$$
\tau=(d u / d y)_{y= \pm 1}
$$

This, using Eq. (36), takes the form $\tau=\left(d u_{0} / d y\right)_{y= \pm 1}+N\left(d u_{1} / d y\right)_{y= \pm 1}$

Similarly, the rate of heat transfer between the fluid and the plate is given by heat flux

$q=-k(\partial T / \partial y)_{y= \pm b}$

where ' $q$ ' is the heat flux. Making Eq. (39) dimensionless using the scale, $\theta=\left(T-T_{0}\right) / \Delta T$ and $N_{u}=q b / k$, we express the heat flux in terms of the Nusselt number $N_{u}$ as

$N_{u}=-(\partial \theta / \partial y)_{y= \pm 1}$

This, using Eq. (20), can be written as

$N_{u}=\left(d \theta_{0} / d y\right)_{y= \pm 1}+N\left(d \theta_{1} / d y\right)_{y= \pm 1}$

Here $\left(d u_{0} / d y\right),\left(d u_{1} / d y\right)$, and $\left(d \theta_{0} d y\right)$ and $\left(d \theta_{1} / d y\right)$ are obtained using the analytical solutions given in section 3 and also computed numerically using Newton quadratic interpolation formula for differentiation. The results obtained are depicted graphically in Fig. 4 and Fig. 5 for skin friction and rate of heat transfer respectively and the conclusions are drawn in the final section 6 .

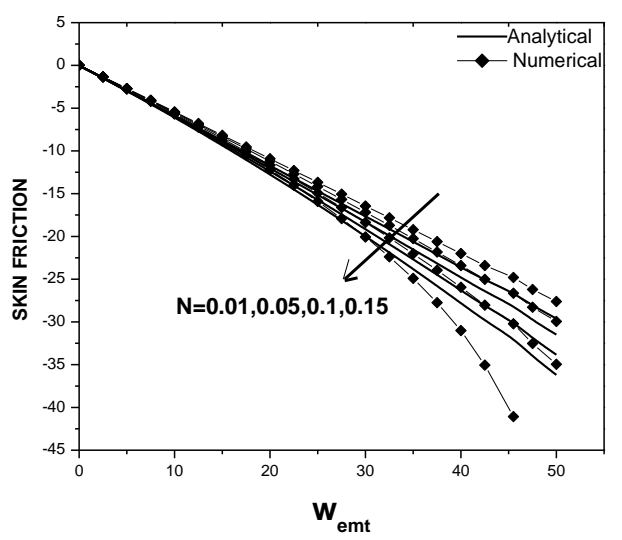

Fig. 4. Skin friction against $\mathrm{W}_{\mathrm{emt}}$ with $\mathrm{Re}=0.5, \mathrm{Pe}=0.7$ and for $\mathrm{N}=0.01$ and 0.1

If ' $\mathrm{m}_{\mathrm{f}}$ ' denotes the dimensionless mass flow rate per unit channel width in the presence of dissipation, then

$m_{f}=\int_{-1}^{1} u d y$

It is of practical Importance to compute the mass flow rate given by Eq. (42) using both analytical and numerical techniques. The analytical solution is obtained using those given in section 3. Similarly the numerical solution is obtained using Simpson's onethird rule for integration. The values obtained from computing the skin friction ' $\tau$ ' given by Eq. (38), the Nusselt number $N_{u}$ given by Eq. (41) and mass flow rate ' $m_{f}$ ' given by Eq. (42) are depicted graphically in Figs. 4-6 respectively and the conclusions are drawn in the final section 6 . 


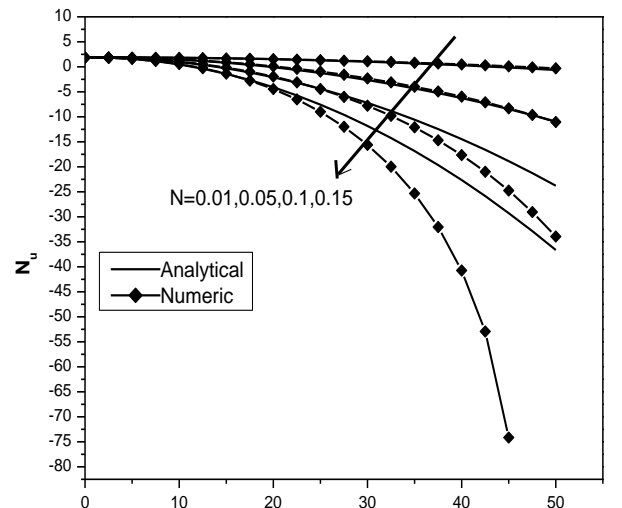

Fig. 5. Rate of heat transfer against $\mathrm{W}_{\mathrm{emt}}$ when $\mathrm{Re}=0.5, \mathrm{Pe}=0.7$ and for $\mathrm{N}=0.01$ to 0.15

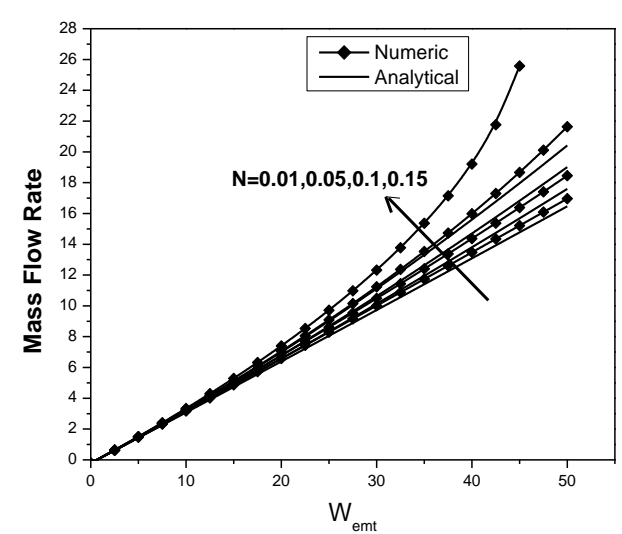

Fig. 6. Mass flow rate against $\mathrm{W}_{\text {emt }}$ with $\mathrm{Re}=0.5$, $\mathrm{Pe}=0.7$ and for $\mathrm{N}=0.01$ to0.15

\section{RESULTS AND DISCUSSION}

The analytical solutions obtained in section 3 for velocity and temperature distributions are computed for a symmetric heating and the results for velocity are depicted graphically in Fig. 2 against $y$ for various values of $W_{e m t}$ with fixed values of $N=0.01, R e=5$, and $P e=7$. From this, we found that the effect of increase in the value of $W_{e m t}$ is to increase the velocity distribution. This implies that the magnetic field augments convection in chiral fluid. Physically this is attributed to the fact that the magnetic field and suction velocity introduce the small scale turbulence in a chiral fluid.

The temperature distribution for various values of $W_{e m t}$ with $N=0.01, R e=5$, and $P e=7$ are drawn in Fig 3 . From this figure it is clear that the temperature distribution also increases with an increase in $W_{e m t}$ and sets up meandering of thermal lines near the hotter plate at $y=1$ implying the thermal boundary layer. Therefore, this increase in the thermal distribution at $y=1$ for different values of $W_{\text {emt }}$ is due to the existence of thermal boundary layer effect at hotter plate $y=1$.

To know the effect of the other parameters namely $N$, $R e$, and $P e$, the velocity and temperature are also computed both analytically and numerically for different values of the $W_{e m t}$ and we found analogous results as given above and hence omitted here. We also observe that the results obtained from the analytical and numerical solutions agree well.

The skin friction ' $\tau$ ' at the cooler plate given by Eq. (38) is computed for various values of $W_{e m t}$ with $R e=5, P e$ $=7, N=0.01$ to 0.1 and the values obtained are depicted graphically in Fig. 4. From this, it is clear that the skin friction decreases with an increase in $W_{\text {emt }}$ but increases with an increase in $N$. Similarly, the rate of heat transfer given by Eq. (41) is computed for various values of $W_{e m t}$, when $R_{e}=5, P e=7, N=0.01,0.05,0.1$ and 0.15 and the results are depicted graphically in Fig. 5. From this figure, we found that $N_{u}$ remains uniform for values of $W_{\text {emt }}$ up to 15 and $N$ up to 0.15 and then decreases with an increase in $W_{e m t}$ but increases with $N$.

The analytical and numerical solutions obtained for mass flow rate given by Eq. (47) are computed for different values of $W_{e m t}$ and for $R e=0.5, P e=0.7$, $N=0.01,0.05,0.1$ and 0.15 and the results are depicted graphically in Fig. 6. From this figure it is clear that the mass flow rate increases with an increase in $\mathrm{W}_{\mathrm{emt}}$ and $N$.

\section{ACKNOWLEDGEMENTS}

This work is supported by ISRO under the research projects no. ISRO/RES/2/338/2007-08 and ISRO/RES/2/335/2007-08. ISRO's financial support to carryout this research is gratefully acknowledged. One of us (Nagaraju) expresses his gratitude to Prof. M.N Channabasappa, Director and Prof. Shivakumariah Principal Siddaganga Institute Technology, Tumkur for their encouragement to continue my research under TEQP.

\section{REFERENCES}

Arago D.F. (1812). Su rune modification remarquable qu'eprouvent les rayons lumineux dans leur passage a tranverse certains corps diaphanes, et sur quelques auters nouveaux phenomenes d'optique, Mem. Inst., 1, 93-134.

Boit, J.B. (1812), emoire sur un nouveau genre d'oscillations que les molecules de la lumiere eprouvent en traversant certains cristaux. Mem. Inst., 1, 372.

Engheta, N. and S. Bassiri (1989). One-and TwoDimensional Dyadic Green's Function in Chiral Media, IEEE Trans on Antennas and Propagation, $37,512-515$.

Engheta, N. and A.R. Mickelson (1982). Transition Radiatin Caused by a Chiral Plate, IEEE Trans on Antennas and Propagation, 30, 1213-1216.

Jaggard, D.L., A.R. Mickelson and C.H. Papas (1979). On Electromagnetic Waves in Chiral Media, Applied Physics, 18, 211-216.

Lakhtakia, A., V.V. Vasundara and K.V. Vijay (1988). Field equations, Huygens,s principle, integral equations, and theorems for radiation and scattering of electromagnetic waves in isotropic chiral media, J. Opt. Soc. Am. A, 175-184. 
N. Rudraiah et al. / JAFM, Vol. 7, No. 1, pp. 35-41, 2014.

Rudraiah, N., M. Vekatachalappa, A. A. Natarajan and T. Basavaraju (2000). Field equations in Chiral Material and Eddy current losses in transformers, Proc. INSA Conference on Math and its applications to Industry, 1-21.

Rudraiah, N., T. Masouka and M.S. Malashetty (1983). Natural convection in a vertical porous layer, Int. Comm. In Heat and Mass Transfer, 10(1), 59-78.

Rudraiah, N., M. Venkatachalappa and M.S. Malashetty (1982). Natural convection through vertical porous stratum, Proc. Indian Acad. Sci. (Math.Sci.), 91(1) 17-37.

Rudraiah N. and S.T. Nagaraj (1977). Natural convection through vertical porous stratum, Int. J. Engg. Sci., 15, 589-600.

\section{APPENDIX}

$A_{1}=-\operatorname{coth} P e, A_{2}=\operatorname{cosechPe}, A_{3}=\left(A_{1}-W_{e m t}\right) / \mathrm{Re}$,

$A_{4}=A_{2} / a_{17}, A_{5}=a_{12}\left(\left(A_{1}-W_{e m t}\right) / \operatorname{Re}+A_{2} a_{13} / a_{16}\right)$,

$A_{6}=\left(\left(\left(-A_{1}+W_{e m t}\right) a_{11}\right) / \operatorname{Re}+\left(A_{2}\left(\cosh P e-a_{11} a_{13}\right) / a_{17}\right)\right.$,

$A_{7}=2 A_{3} A_{5} /(P e-\mathrm{Re}), A_{8}=\left(2 A_{4} A_{5} P_{e}\right) /(P e+\mathrm{Re})$,

$A_{9}=\left(A_{5}^{2} \mathrm{Re}\right) /(2 P e-4 \mathrm{Re})$

$A_{10}=\left(-A_{3}^{2} \cosh P e\right) / P e+A_{4} 2 \cosh P_{e}$

$+A_{3}\left(2 A_{4} \operatorname{coth} \mathrm{P} e-\left(-A_{5} \cosh P e \sinh \mathrm{Re}\right) /(P e-\mathrm{Re})\right)$,

$A_{11}=-A_{4}^{2} / 2+\left(A_{3}^{2} \operatorname{coth} P e\right) / P e-$

$\left(A_{5}^{2} \mathrm{Recosh} P e \sinh (P e-2 \mathrm{Re})\right) / 2(P e-2 \mathrm{Re})$

$-\left(-2 A_{4} A_{5} P e \cosh P e \sinh \mathrm{Re}\right) /(P e+\mathrm{Re})-$

$\left(2 A_{3} \cosh P_{e}\left(A_{4}\left(P_{e}+R_{e}\right)+A_{5} \sinh \left(P_{e}-R_{e}\right)\right)\right) /\left(P_{e}+R_{e}\right)$

$a_{1}=2 P e \operatorname{Re}^{2}, a_{2}=\left(a_{12} / a_{3}\right)\left(e^{\mathrm{Re}} \sinh 2 P e-\sinh (2 P e-\mathrm{Re})\right.$,

$a_{4}=\left(a_{11} a_{13}\right) / a_{14}, a_{5}=\left(a_{11} \sinh (P e+\operatorname{Re})\right) / a_{14}$,

$a_{6}=\operatorname{Re}-2 P e(P e-\operatorname{Re})$

$a_{11}=\operatorname{coth} \operatorname{Re} a_{12}=\cosh \operatorname{Re}, a_{13}=\sinh P e$,

$a_{14}=P e^{2}+\operatorname{Re} P e, a_{15}=P e-P e \operatorname{Re}$,

$a_{16}=P e^{2}(P e-\operatorname{Re}), a_{17}=P_{e}\left(P_{e}-R_{e}\right)$. 